\title{
Molecular consequences of SOD2 expression in epigenetically silenced pancreatic carcinoma cell lines
}

\author{
EM Hurt ${ }^{*}$, , SB Thomas², B Peng ${ }^{3}$ and WL Farrar ${ }^{*, 1}$ \\ 'Cancer Stem Cell Section, Laboratory of Cancer Prevention, Center for Cancer Research, National Cancer Institute at Frederick, National Institutes of \\ Health, Frederick, MD 21702, USA; ${ }^{3}$ Basic Research Program, SAIC-Frederick Inc., National Cancer Institute at Frederick, Frederick, MD 21702, USA; \\ ${ }^{3}$ School of Dental Science, University of Melbourne, Melbourne, Victoria, Australia
}

Manganese superoxide dismutase (SOD2) is an enzyme that catalyses the dismutation of superoxide in the mitochondria, leading to reduced levels of reactive oxygen species. Reduced expression levels of SOD2 have been shown to result in increased DNA damage and sod2 heterozygous mice have increased incidences of cancer. It has also been shown that SOD2 expression is lost in pancreatic cell lines, with reintroduction of SOD2 resulting in decreased rate of proliferation. The mechanism of decreased SOD2 expression in pancreatic carcinoma has not been previously determined. We demonstrate, through sodium bisulphite sequencing, that the sod2 locus is methylated in some pancreatic cell lines leading to a corresponding decrease in SOD2 expression. Methylation can be reversed by treatment with zebularine, a methyltransferase inhibitor, resulting in restored SOD2 expression. Furthermore, we demonstrate that sensitivity of pancreatic carcinoma cell lines to 2-methoxyestradiol correlates with SOD2 expression and SOD2 modulation can alter the sensitivity of these cells. Using both genomics and proteomics, we also identify molecular consequences of SOD2 expression in MIA-PaCa2 cells, including dephosphorylation of VEGFR2 and the identification of both SOD2-regulated genes and transcription factors with altered binding activity in response to SOD2 expression.

British Journal of Cancer (2007) 97, I I 16 - I | 23. doi: I 0. I 038/sj.bjc.6604000 www.bjcancer.com

Published online 25 September 2007

(c) 2007 Cancer Research UK

Keywords: superoxide dismutase 2; pancreatic neoplasms; proteomics; genomics; epigenetic process

Free radicals are, arguably, considered among the most potent, ubiquitous, endogenous mutagens generated by normal physiological processes (Hussain et al, 2003). Among various molecular forms of free radicals, reactive oxygen species (ROS) have been the most diligently studied. Generated by all cells as a by-product of oxidative metabolism and inflammatory processes, ROS are indicted in inducing cellular aging (Melov, 2000), cancer, and a variety of other pathophysiological conditions. Alterations in macromolecular functions are understandable in view that ROS may target DNA, proteins, RNA, and lipids. Due to the deleterious potential of free radicals, mechanisms have evolved to protect cells from ROS, including antioxidant scavengers, enzymes, and repair mechanisms.

Cancer, in particular, arises from a combination of potential genetic mutations, and possibly epigenetic changes, altering the cells' normal proliferative and apoptotic programming. Within the last few years, changes in expression patterns of enzymes that

* Correspondence: EM Hurt, Laboratory of Cancer Prevention, National Cancer Institute at Frederick, 1050 Boyles Street, Building 560, Room 2I-8I, Frederick, MD 21702, USA; E-mail: hurte@ncifcrf.gov or

WL Farrar, Laboratory of Cancer Prevention, National Cancer Institute at Frederick, 1050 Boyles Street, Building 560, Room 21 -78, Frederick, MD 21702, USA; E-mail: farrar@ncifcrf.gov

Received 2 April 2007; revised 14 August 2007; accepted 29 August 2007; published online 25 September 2007 stand as guardians for ROS have been implicated in the possible progression or initiation of some cancers. Considered among the earliest pre-neoplastic changes in prostate cancer is the epigenetic silencing by promoter CpG hypermethylation of the glutathione $S$-transferase $\pi$ gene (Lin et al, 2001; Nelson et al, 2001). Thus, speculating that a reduction in the ability to manage ROS leads to further mutation and advancement of the neoplastic process.

Among the enzymes produced by cells that manage ROS, manganese superoxide dismutase (SOD2), the form of SOD found in mitochondria, has received considerable attention with regard to cancer (Liu et al, 2004). The main function of SOD2 is to convert superoxide anion into hydrogen peroxide $\left(\mathrm{H}_{2} \mathrm{O}_{2}\right)$, which is subsequently converted to water by catalases. Decreased expression of SOD2 has been noted, initially, in pancreatic carcinoma cells lines (Cullen et al, 2003). More recently, reduced expression of SOD2 has been seen in multiple myeloma cells (Hodge et al, 2005b), androgen-independent prostate cancer (Best et al, 2005; Venkataraman et al, 2005), and invasive breast carcinoma (Soini et al, 2001). Overexpression of SOD2 in deficient cell lines has reduced the proliferation of pancreatic carcinoma cells (Weydert et al, 2003; Ough et al, 2004), multiple myeloma cells (Hodge et al, 2005b), glioma cells (Zhong et al, 1997), squamous oral carcinoma cells (Liu et al, 1997), and prostate carcinoma cells (Li et al, 1998b; Zhong et al, 2004; Venkataraman et al, 2005). Transgenic overexpression of SOD2 also results in increased resistance to chemical carcinogenesis as compared to wild-type mice (Zhao et al, 2001). While homozygous sod2 knockout mice die within 
weeks, heterozygous $+I-$ mice have accelerated tumour development, particularly lymphomas (Van Remmen et al, 2003). Taken together, these data have raised the compelling issue that, in addition to the effects of SOD2 as a guardian against ROS, SOD2 may phenotypically act as a tumour suppressor (Cullen et al, 2003).

While the phenotypic effects of diminished and/or overexpressed SOD2 have been reported for various tumour cell lines, the explanation for reduced expression has only recently emerged. Polymorphisms of the sod2 promoter have been suggested as one possible mechanism for reduced expression of SOD2 in certain cell lines (Xu et al, 1999). More recently, we have found that the sod2 promoter is methylated in some multiple myeloma cells and diminished expression can be reversed by the methyltransferase inhibitor zebularine (Hodge et al, 2005a), suggesting that repression of SOD2 may occur at the level of epigenetic regulation. In addition to understanding the relative mechanisms of SOD2 expression levels in cancer cells, little is known about the consequences of reduced SOD2 on the signal transduction and transcriptional processes that control cancer cell viability and growth. Some data have emerged suggesting that levels of SOD2 have effects on AP-1 and NF- $\kappa \mathrm{B}$ activities, in which overexpression experiments diminish the activities of these two important transcription factors (Kiningham and St Clair, 1997; Li et al, 1998a).

Here, using a series of pancreatic carcinoma cell lines, we show that the relative expression patterns of SOD2 are inversely related to the methylation density status of the sod 2 promoter. Hypermethylation of $\mathrm{CpG}$ sites was rapidly reversed by the methyltransferase inhibitor zebularine, restoring SOD2 levels. The epigenetic silencing of sod2 revealed a particular Achille's heel of pancreatic carcinoma cells with low SOD2, making the cells particularly susceptible to the apoptotic effects of 2-methoxyestradiol (2ME2), an oxidative burst agent.

To examine the more global consequences of SOD2 expression on the cellular processes of pancreatic carcinoma, we have employed a gestalt approach of examining how SOD2 affects vital processes of signal-transduction proteins, transcription factor activation, and gene regulation. Using reverse-phase antibody arrays for signal proteins, transcription factor arrays and gene expression microarrays, we have identified some of the major aspects in which SOD2 governs signal transduction and gene expression in pancreatic carcinoma cells.

\section{MATERIALS AND METHODS}

\section{Genomic DNA isolation, bisulphite modification, and PCR amplification}

Genomic DNA was isolated using Qiagen's DNA isolation kit (Qiagen). DNA was EcoRI digested, denatured with $0.3 \mathrm{M} \mathrm{NaOH}$ for $15 \mathrm{~min}$ at $37^{\circ} \mathrm{C}$, and modified with $3.1 \mathrm{M}$ sodium bisulphite and $0.5 \mathrm{~mm}$ hydroquinone. Modified DNA was purified using the QIAquick DNA extraction kit (Qiagen, Valencia, CA, USA) and treated with $0.3 \mathrm{M} \mathrm{NaOH}$ for $15 \mathrm{~min}$ at $37^{\circ} \mathrm{C}$ to complete the modification. Nested PCR was used to amplify the modified DNA fragments. The first set of primers for the sod2 gene promoter region (Genbank accession no. L34157) was $5^{\prime}$-GtAtttTtAGGGG [C/t]GGAt[C/t]GGAGGtAGGGtTT-3' (sense, nt 1896-1927) and $5^{\prime}$-CCAaaCCC[a/G]aTaC[a/G]aCCACTaTC[a/G]CCATTaC-3' (antisense, nt 2520-2490), and the second set of primers was $5^{\prime}$-GGGt [C/t]GTAttAAtTttA[C/t]GGGGGtAGGGGt-3' (sense, nt 1929-1958) and $5^{\prime}$-AaCCCCTTaCCCCTTaaaaC[a/G]TaACC[a/G]aaTCCC-3' (antisense, nt 2468-2436), where lower case letters represent bisulphiteconverted nucleotides. PCR cycles were as follows: $95^{\circ} \mathrm{C}$ for $30 \mathrm{~s}$, followed by 35 cycles at $95^{\circ} \mathrm{C}$ for $30 \mathrm{~s}, 55^{\circ} \mathrm{C}$ for $30 \mathrm{~s}, 72^{\circ} \mathrm{C}$ for $2 \mathrm{~min}$, and final extension at $72^{\circ} \mathrm{C}$ for $10 \mathrm{~min}$. The amplified DNA fragment of expected size was cloned into pCR4-TOPO TA cloning vector (Invitrogen, Carlsbad, CA, USA). Ten individual clones for each cell line were sequenced.

\section{Construction of SOD2 expression and shRNA retroviruses}

Adenoviral SOD2 construct (kind gift of JJ Cullen, University of Iowa) was EcoRI digested and sod2 cDNA was cloned into the retroviral vector pLZRS-BMN-eGFP containing an IRES and eGFP cDNA. For overexpression experiments, the control was the empty eGFP vector. The oligos used for shRNA knockdown of sod2 are as follows: 5'-GATCCCGGGGTTGGCTTGGTTTCAATATTCAAGAGA TATTGAAACCAAGCCAACCCCTTTTTA- $3^{\prime}$ and $5^{\prime}$-AGCTTAAAA AGGGGTTGGCTTGGTTTCAATATCTCTTGAATATTGAAACCAA GCCAACCCCGG-3'. The oligos were annealed and cloned into the BglII/HindIII sites of pRetroSuper (Brummelkamp et al, 2002).

\section{Generation of stable cell lines with altered SOD2 expression levels}

A bicistronic retrovirus carrying sod2 cDNA and eGFP or an empty vector (control) was used to create the overexpression cell lines. For knockdown of SOD2, the shSOD2-pRetroSuper or empty pRetroSuper was used to infect pancreatic cell lines. In both instances, the Phoenix Amphotropic cells (kind gift of Gary Nolan, Stanford University) were transfected using FuGENE 6 (Roche Diagnostics, Indianapolis, IN, USA), according to manufacturer's instructions. Two days following transfection, $3 \mathrm{ml}$ of viral supernatant was used to infect $1-3 \times 10^{6}$ pancreatic cells. For overexpression of SOD2, cells were flow sorted for high GFP expression, whereas for shSOD2 and control cells were selected with $1 \mu \mathrm{g} \mathrm{ml}^{-1}$ puromycin $48 \mathrm{~h}$ post infection. Expression of SOD2 was determined by western blot (anti-SOD2, ab16954; Abcam, Cambridge, MA, USA).

\section{Determination of superoxide dismutase activity in cell lines}

Superoxide dismutase activity was measured using Calbiochem's Superoxide Dismutase Assay Kit II according to manufacturer's directions. Briefly, $2 \times 10^{6}$ cells were sonicated in $20 \mathrm{mM}$ HEPES buffer containing $1 \mathrm{~mm}$ EGTA, $210 \mathrm{~mm}$ mannitol, and $70 \mathrm{~mm}$ sucrose.

Ten microlitres of lysate was mixed with $200 \mu \mathrm{l}$ of radical detector (tetrazolium salt) containing $1 \mathrm{~mm}$ potassium cyanide to inhibit $\mathrm{Cu} / \mathrm{Zn}$-SOD and $20 \mu \mathrm{l}$ xanthine oxidase. The reaction was incubated at room temperature for $20 \mathrm{~min}$ and read in a Genios (Tecan, Palm Springs, CA, USA) plate reader at $450 \mathrm{~nm}$. One unit of SOD activity is defined as the amount of enzyme needed to exhibit $50 \%$ dismutation of the superoxide radical.

\section{Measurement of proliferation by MTT assay}

Cells were seeded in triplicate at $2 \times 10^{4}$ per well in a 96-well plate. The 3-(4,5-dimethylthiazol-2-yl)-2,5-diphenyltetrazolium bromide (MTT) assay was performed in accordance with the manufacturer's instructions (Chemicon, Temecula, CA, USA). Briefly, following addition of MTT, the cells were incubated at $37^{\circ} \mathrm{C}$ for $2 \mathrm{~h}$. The precipitate was resuspended in isopropanol containing $0.04 \mathrm{~N}$ hydrochloric acid and read immediately using a Genios (Tecan) plate reader at $570 \mathrm{~nm}$.

\section{2-Methoxyestradiol treatment and comet assay}

One million cells were treated with $3 \mu \mathrm{M} 2 \mathrm{ME} 2$ for $48 \mathrm{~h}$. DNA damage was assessed using the CometAssay kit (Trevigen, Gaithersburg, MD, USA). Briefly, 500-1000 cells were embedded in $1.0 \%$ LMAagrose on a Cometslide and lysed $(2.5 \mathrm{M}$ sodium 
chloride, $100 \mathrm{~mm}$ EDTA, $\mathrm{pH}$ 10, $1 \mathrm{~mm}$ Tris base, $1 \%$ sodium lauryl sarcosinate, and $1 \%$ Triton $\mathrm{X}-100)$. The slides were then electrophoresed in alkaline electrophoresis solution $(300 \mathrm{~mm}$ $\mathrm{NaOH}$ and $1 \mathrm{~mm}$ EDTA) for $30 \mathrm{~min}$ at $300 \mathrm{~mA}$. Cells were stained with SYBR green and viewed by fluorescence microscopy. The comet tail moment for 50 nuclei per treatment was measured using ImageJ software (http://rsb.info.nih.gov/nih-image).

\section{Oligonucleotide expression microarrays}

Oligonucleotide microarrays were prepared by the National Cancer Institute's microarray core facility, using Operon's Human version 3.0 oligo set (Operon Biotechnologies, Huntsville, AL, USA) printed on UltraGAPS slides (Corning, Corning, NY, USA). This set contains 34580 oligos, representing approximately 25000 unique genes. Total RNA $(20 \mu \mathrm{g})$ was labelled in a reverse transcription (RT) reaction with an oligo-dT primer and either Cy3-dUTP or Cy5-dUTP. Following the RT reaction, the RNA was hydrolysed by incubation with $\mathrm{NaOH}$. Probes were purified with a Microcon YM-30 column (Millipore, Billerica, MA, USA). Microarrays were prehybridised in $5 \times$ SSC, $0.1 \%$ SDS, and $1 \%$ BSA for $1 \mathrm{~h}$ at $42^{\circ} \mathrm{C}$, washed in distilled water and then in $100 \%$ ethanol, and air dried. Hybridisation of mixed $\mathrm{Cy}-3-$ and $\mathrm{Cy}$-5-labelled probes was performed in $50 \%$ formamide, $10 \times$ SSC, and $0.2 \%$ SDS overnight at $42^{\circ} \mathrm{C}$. After hybridisation, slides were washed in decreasing concentrations of SSC $(2 \times$ SSC $+0.1 \%$ SDS, $1 \times$ SSC, $0.2 \times$ SSC). Arrays were scanned in a GenePix 4000B scanner and analysed using GenePix Pro (Molecular Devices, Sunnyvale, CA, USA). Data analysis was preformed using Cluster and TreeView, offered by Michael B Eisen as freeware (http://rana.lbl.gov/ EisenSoftware.htm).

\section{Reverse-phase protein arrays}

Protein lysates were prepared for printing on reverse-phase protein arrays (RPAs) by lysis of 10000 cells in T-PER (Pierce, Rockford, IL, USA). Five two-fold serial dilutions were made from each lysate. RPAs were printed as previously described (Paweletz et al, 2001). Briefly, arrays were printed on nitrocellulose-coated glass slides (FAST Slides, Whatman, Florham Park, NJ, USA) using a GMS 417 arrayer (Affymetrix, Santa Clara, CA, USA). Each array was incubated with primary antibody and detected using the catalysed signal amplification system (Dako, Carpinteria, CA, USA) and an Autostainer Universal Staining System (Dako). Slides were blocked with I-block (Tropix, Bedford, MA, USA), incubated with primary and secondary antibodies, streptavidin-biotin complex, biotinyl tyramide, streptavidin-peroxidase, and developed with 3,3'-diaminobenzidine tetrahydrochloride chromogen with washing between all steps. Total protein was determined by staining arrays with AuroDye Forte (GE Healthcare, Piscataway, NJ, USA). The arrays were scanned with an Epson flat-bed scanner (Epson, Long Beach, CA, USA) and quantitated using MicroVigene with the dilution curve module (ViGene Tech, Carlisle, MA, USA). For each sample, the slope of the regression line best fitting the linear range of the dilution curve was used to determine relative protein expression. All experimental values were normalised to total protein.

\section{DNA/protein arrays}

Panomic's DNA/protein Combo arrays containing 345 consensus binding sites were hybridised according to manufacturer's instructions. Briefly, nuclear protein was extracted using Panomic's Nuclear Protein extraction kit following manufacturer's instructions. Fifteen micrograms of each protein lysate was used in the binding reaction. Following clean-up, the probe was hybridised at $45^{\circ} \mathrm{C}$ overnight. Data were quantitated using ImageQuant (Amersham Biosciences), and a binding site was considered to be regulated only if it showed reciprocal regulation in the overexpression and the shRNA cell lines.

\section{RESULTS}

Sod2 is methylated in some pancreatic cell lines leading to decreased expression

Several reports have demonstrated decreased expression of SOD2 in pancreatic carcinoma (Su et al, 2002; Cullen et al, 2003) without determining the underlying cause. To determine if decreased SOD2 expression could be the result of hypermethylation of the promoter, we performed sodium bisulphite sequencing on four pancreatic cell lines. We observed a high methylation density of the sod2 promoter in MIA-PaCa2, intermediate methylation densities in AsPc-1 and BxPc-3, and a low density in Capan-1 (Figure 1A). The degree of methylation was inversely correlated with protein expression levels (Figure 1B).

\section{Methylation of the sod 2 promoter can be reversed with zebularine resulting in re-expression of SOD2}

To further substantiate the finding of hypermethylation of the sod2 promoter, we treated the three cell lines with zebularine (Driscoll et al, 1991), a methyltransferase inhibitor (Zhou et al, 2002), and determined methylation by sodium bisulphite sequencing (Figure 1C). Treatment with zebularine nearly eliminated methylation of the sod2 promoter in both MIA-PaCa2 and AsPc-1. In $\mathrm{BxPc}-3$, methylation was minimally decreased and showed a more limited pattern of demethylation with three regions showing a greater extent of demethylation than the remaining areas. Capan-1 showed no significant changes in methylation densities. To determine if the demethylation in response to zebularine treatment lead to a corresponding increase in expression of SOD2, we performed a western blot following zebularine treatment. Both MIA-PaCa2 and AsPc-1 showed a significant increase in SOD2 protein levels (Figure 1D). There was no increase in SOD2 expression in both BxPc-3 and Capan-1 upon treatment with zebularine (Figure 1D) consistent with the lesser extent of demethylation.

\section{SOD2 expression levels predict 2ME2 sensitivity that can be ameliorated by introduction of SOD2}

Pancreatic carcinomas have been shown to be sensitive to 2ME2 (Schumacher et al, 1999; Qanungo et al, 2002). The mechanism of 2ME2-induced apoptosis involves mitochondrial-dependent pathways (Qanungo et al, 2002) as well as ROS (Chauhan et al, 2003). With diminished capacity to detoxify superoxide radicals, cells with silenced SOD2 should exhibit higher sensitivity to 2ME2. Using the four pancreatic carcinoma cell lines with variable SOD2 expression, we examined 2ME2 sensitivity in an MTT assay (Figure 3A). Capan-1, which has the highest endogenous expression of SOD2, was the least sensitive to 2ME2, whereas MIA-PaCa2, which has the lowest endogenous expression of SOD2, showed the highest sensitivity to 2ME2 (Figure 2A). MIA-PaCa2 cells are sensitive to 2ME2 (Schumacher et al, 1999), and the inhibition of MIA-PaCa2 measured in the MTT assay is similar to the $42 \%$ of apoptotic nuclei in MIA-PaCa2 following 2ME2 treatment for $48 \mathrm{~h}$ reported by Qanungo et al (2002). To test whether SOD2 expression itself could decrease sensitivity to $2 \mathrm{ME2}$, we engineered MIA-PaCa2 cells with altered levels of SOD2. We demonstrate that these cell lines show both the expected level of expression and enzymatic activity (Supplementary Figure 1). SOD2 overexpression in MIA-PaCa2 leads to a slight reduction in sensitivity to 2ME2, whereas SOD2 knockdown showed a slight increase in sensitivity (Figure 2B). 


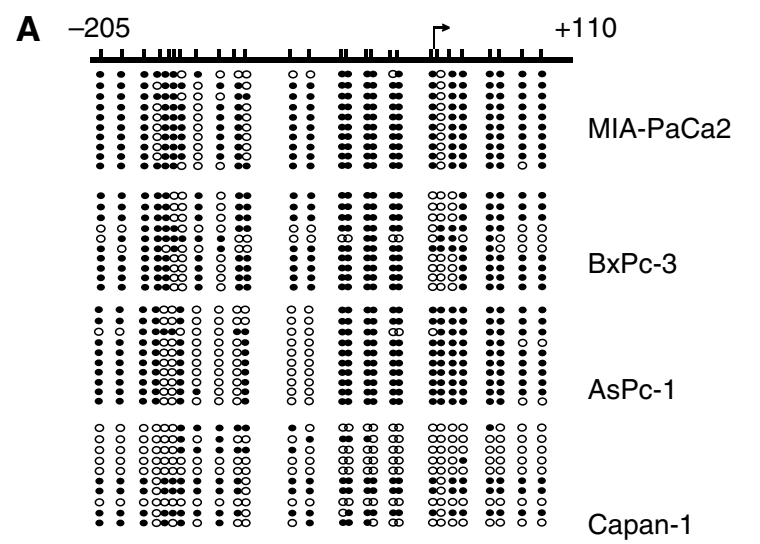

B

C -205

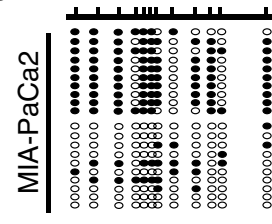

$+110$
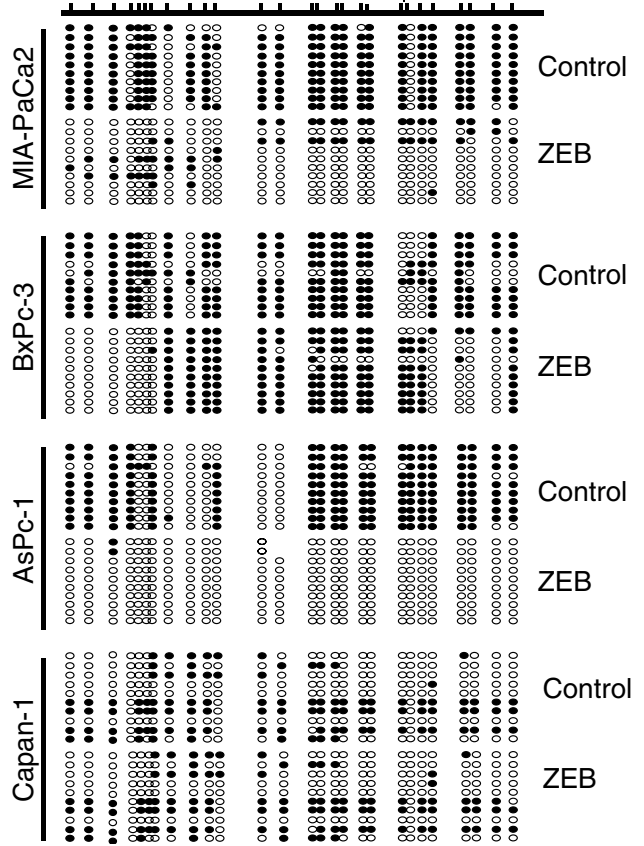

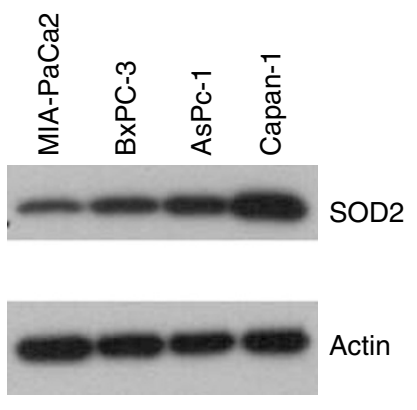

D
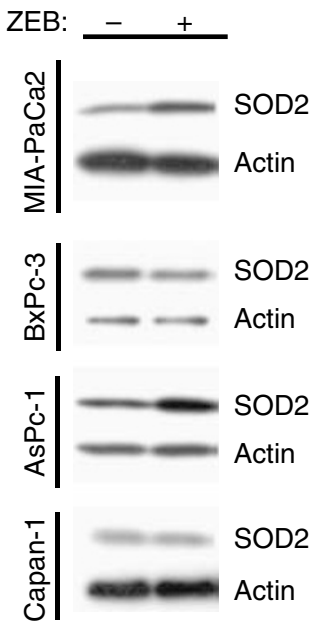

Figure I The sod2 promoter is hypermethylated in some pancreatic carcinomas that is inversely correlated with protein levels. (A) The methylation status of the sod2 promoter was examined by sodium bisulphite sequencing in four pancreatic carcinomas. All CpG dinucleotides are represented by either an open circle $(O)$ to indicate unmethylated cytosines or a filled circle $(\mathbf{O})$ to indicate a methylated cytosine. (B) Protein levels of SOD2 were determined by western blot. The level of SOD2 expression is inversely correlated with the extent of hypermethylation, with Capan-I showing the lowest level of methylation and the highest level of expression. Actin serves as a loading control. (C) Sodium bisulphite sequencing of three cell lines that showed hypermethylation of the sod2 promoter following treatment with a methyltransferase inhibitor, zebularine, for $72 \mathrm{~h}$. (D) Protein expression levels of SOD2 in pancreatic carcinoma lines following zebularine treatment.

\section{Treatment with 2ME2 leads to DNA damage and apoptosis of pancreatic cell lines}

Since 2ME2 induces ROS and PARP activation in pancreatic cell lines (Qanungo et al, 2002), we tested whether or not 2ME2 induced DNA damage. Following treatment with 2ME2, there was significant DNA damage induced in both MIA-PaCa2 and BxPc-3 (Figure 2C). MIA-PaCa2 showed a nearly three-fold increase in tail length following treatment with $2 \mathrm{ME} 2$ as compared to control (Figure 2C), whereas BxPc-3 showed an approximate two-fold increase in tail length following 2ME2 treatment (Figure 2C). Both AsPc-1 and Capan-1 showed little to no tailing following treatment with $2 \mathrm{ME} 2$, indicating that $2 \mathrm{ME} 2$ did not lead to DNA damage in these cell lines (Figure 2C and D). Capan-1 has the highest expression of SOD2 and therefore the largest capacity to clear superoxide radicals, which may account for the limited DNA damage by $2 \mathrm{ME} 2$. While BxPc-3 shows a modest level of SOD2 expression, there was no tailing in response to $2 \mathrm{ME} 2$, which may be due to increased expression of other ROS-scavenging enzymes or better DNA repair mechanisms. While these results suggest that SOD2 expression can reduce 2ME2-induced DNA damage, we sought to directly determine if SOD2 expression alone could ameliorate the effects of 2ME2. Therefore, we performed a comet assay on MIA-PaCa2 with and without overexpression of SOD2. The overexpression of SOD2 in MIA-PaCa2 substantially lessened the extent of DNA damage incurred during treatment with 2ME2, whereas treatment of the shSOD2 MIA-PaCa2 cells resulted in increased damage (data not shown).

\section{Gene expression changes result from the overexpression and knockdown of SOD2}

To address the changes in gene expression that result from SOD2 overexpression, we performed oligonucleotide arrays on MIA$\mathrm{PaCa} 2$ with SOD2 overexpression and knockdown. All genes 


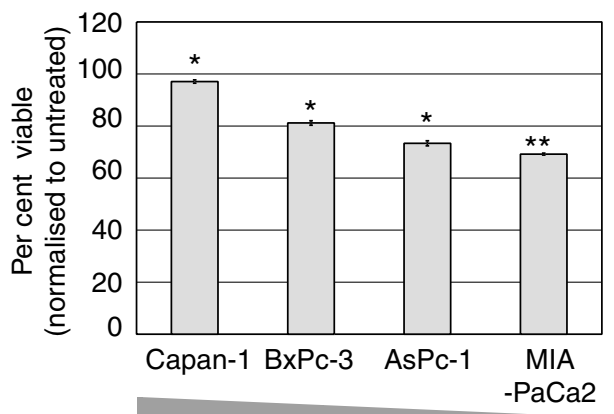

Relative endogenous SOD2 expression

C

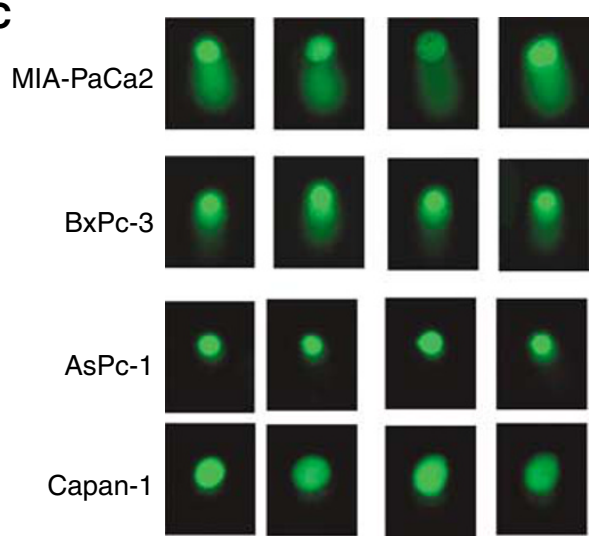

B

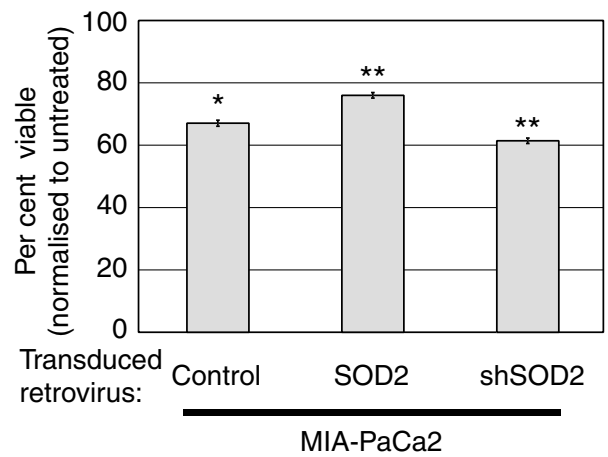

D

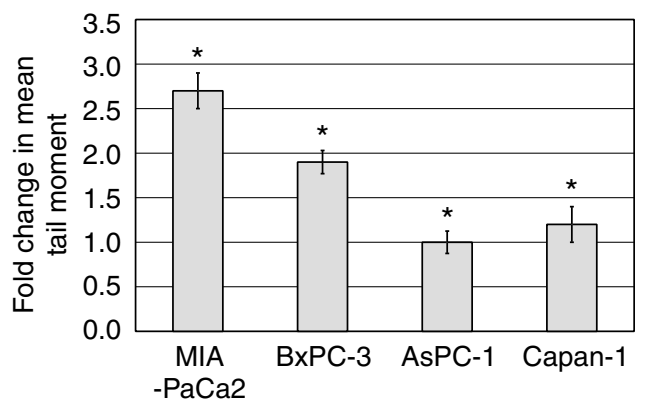

Figure 2 SOD2 protein levels modulate sensitivity to 2-methoxyestradiol and DNA damage. (A) Sensitivity to 2ME2 is inversely correlated with SOD2 expression levels. Following treatment with $3 \mu \mathrm{M} 2 \mathrm{ME2}$ for $48 \mathrm{~h}$, an MTT assay was performed in triplicate. The number of viable cells following $2 \mathrm{ME2}$ treatment is shown as a per cent of the number of viable of vehicle-treated cells. $* P \leqslant 0.05, * * P<0.001$. A representative of three experiments is shown. (B) Determination of sensitivity to 2 ME2 was performed on MIA-PaCa2 cell lines with engineered SOD2 levels in triplicate by MTT. A representative of three experiments is shown. The number of viable cells following 2ME2 treatment is shown as a per cent of the number of viable of vehicle-treated cells. Increased expression of SOD2 results in decreased sensitivity, whereas decreased SOD2 levels result in increased sensitivity. *P $P 0.05$, **P $<0.00$ I. (C) DNA damage was determined by comet assay following treatment with 2ME2. Four representative nuclei for each cell lines are shown. (D) The extent of DNA damage following 2ME2 was determined by measuring the length of the tail moment of 50 nuclei. The fold-over-untreated mean tail moment length is shown where an asterisk indicates a $P$-value $\leqslant 0.05$.

showing at least a two-fold change in expression in three of the four overexpression experiments and an inverse change in the knockdown experiment are shown in Figure 3A. There were 33 genes that showed a decrease in expression in the overexpression experiments by the preceding criteria but no genes showing an increase in expression by these same criteria. These genes are involved in many different cellular processes, including calcium homeostasis (fstl5, man1a1, efhd1, prkacb, and kcnip3), adhesion ( $p s c d b p$ and spon1), GTP metabolism (tbc1d14 and arhgap20), and steroid metabolism (wwox and ugt2b7).

\section{Signalling pathways showing altered activity with SOD2 expression}

ROS has been shown to alter phosphorylation states of various proteins, especially tyrosine phosphorylation. In order to investigate the role of ROS on the phosphoproteome, we performed RPAs with lysates from MIA-PaCa2 containing altered SOD2 levels. Using these arrays, we identified a decrease in the phosphorylation of VEGFR2 when SOD2 was overexpressed (Figure 3B). Other reports have shown modification of VEGFR2 in response to various ROS-inducing agents, including $\mathrm{H}_{2} \mathrm{O}_{2}$ (Gonzalez-Pacheco et al, 2006), which is presumably increased in SOD2-deficient cell lines. In contrast, there was no change in the phosphorylation status of EGFR, STAT3, c-abl, or p-src detected using our RPAs (Supplementary Table 1).

\section{Changes in transcription factor binding as a result of SOD2 expression}

In recent years, there has been considerable evidence that ROS leads to changes in transcription factor binding. In order to determine transcription factors that have altered DNA binding as a result of SOD2 expression, we performed Panomic's Protein/DNA array that examines the binding activities of 354 transcription factor binding sites. The experiment was conducted using nuclear extracts from control MIA-PaCa2 cells, MIA-PaCa2 overexpressing SOD2, as well as MIA-PaCa2 with SOD2 knockdown, and transcription factors showing reciprocal changes in overexpression and SOD 2 knockdown are summarised in Table 1. Consistent with the gene expression data, there were more transcription factors identified that showed a decrease in binding upon SOD2 overexpression. Among those showing the greatest decrease in binding activity were Brn-3, Myc/Mad, and TCF/LEF.

\section{Identification of cognate binding sites for regulated transcription factors in the promoters of regulated genes}

With the identification of both transcription factors showing altered DNA binding upon SOD2 expression and genes regulated by SOD2, we sought to identify cognate binding sites for the regulated transcription factors in the promoters of the regulated genes. Of the 32 genes identified as being downregulated by SOD2 expression (Figure 3), promoter regions were found for 30 of them 
A
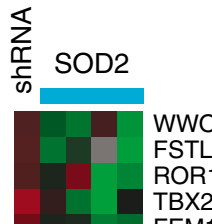

WWOX

ROR1

TBX22

CCDC94

MAN1A1

ATP1A2

SUV39H2

TBC1D14

EFHD1

ARHGAP24

GRIK5

PRKACB

GPHN

PSCDBP

HBB

UGT2B7

CD1A

ABI2

$\mathrm{ABI} 2$

LCT
PTPRS

JPH4

KCNAB1

ARHGAP20

B

ARHGAP

KCNIP3
FNDC3A

ASPA

MAMDC2

SPON1

SPON

CYTL1
CEACAM4

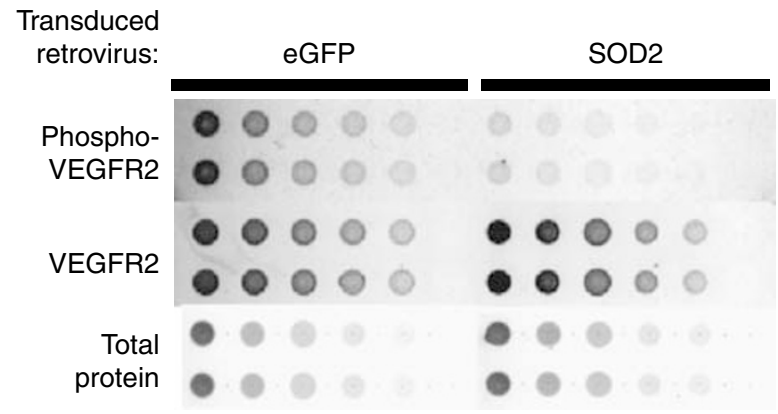

Neat 1:2 1:4 1:81:16 Neat 1:2 1:4 1:81:16

Fold relative expression

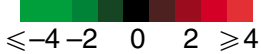

Figure 3 SOD2 expression in MIA-PaCa2 cells results in downregulation of several genes and phosphorylation of VEGFR2. (A) Oligonucleotide arrays were performed on SOD2-engineered MIA-PaCa2 cell lines. All named genes showing at least a two-fold downregulation in two of three SOD2 overexpression experiments as well as an increase in the shSOD2 experiment are shown. (B) Reverse-phase protein arrays were performed to determine differences in the phosphoproteome following manipulation of SOD2 protein levels. Phosphorylation of VEGFR2 is decreased in SOD2-overexpressed MIA$\mathrm{PaCa} 2$ cells. Total VEGFR2 expression remains unchanged between the vector-only control and the SOD2-overexpressed cells, with total protein shown as the loading control.

Table I Transcription factors with modulated DNA binding activities in SOD2-altered MIA-PaCa2 cells

\begin{tabular}{lcc} 
Transcription factor & Fold change SOD2 & Fold change shSOD2 \\
\hline Transcription factors with decreased DNA binding upon infection with SOD2 retrovirus \\
Bm-3 & -10.7 & 1.2 \\
C/EBP & -7.5 & 3.6 \\
NPAS2 & -6.1 & 1.9 \\
Myc-Max & -4.5 & 5.6 \\
LyF-I & -3.7 & 2.0 \\
TCF/LEF & -3.1 & 1.7 \\
NRF-I & -2.8 & 2.6 \\
WTI & -2.5 & 1.8 \\
TCE & -2.1 & 4.6 \\
LRI & -2.1 & 4.4 \\
GATA-2 & -1.8 & 2.0 \\
SPI & -1.6 & 1.5
\end{tabular}

Transcription factors with increased DNA binding upon infection with SOD2 retrovirus

$\begin{array}{lll}\text { Pax-6 } & 2.3 & -1.7 \\ \text { PCF } & 2.6 & -1.7\end{array}$

SOD2 = manganese superoxide dismutase. Transcription factors with altered binding activity in response to engineered SOD2 expression levels in MIA-PaCa2 cells. Transcription factor binding activity was determined by Panomic's DNA/Protein Combo array that profiles the binding activity of 390 transcription factors. Transcription factors that showed reciprocal binding patterns with SOD2 overexpression and knockdown are shown. using Genomatix's Gene2Promoter database. The promoter regions were scanned for binding sites of the downregulated transcription factors listed in Table 2 using Genomatix's MatInspector. Of the 30 promoters searched, binding sites were identified in 25 of them. Table 2 summarises these results.

\section{DISCUSSION}

The generation of ROS is a by-product of several cellular processes, including electron transport (Cadenas, 1989). The elimination of ROS is handled in the cell by several antioxidant enzymes, including superoxide dismutases, which convert the superoxide radical to $\mathrm{H}_{2} \mathrm{O}_{2}$. The $\mathrm{H}_{2} \mathrm{O}_{2}$ then gets converted into water and molecular oxygen by peroxidases. The perturbation of any of these processes can result in the build up of deleterious ROS in the cell. ROS is implicated in the pathology of several cancers, including pancreatic carcinoma (Cullen et al, 2003). Therefore, we sought to better understand the role of ROS in pancreatic carcinoma. In particular, we were interested in the effects that the manipulation of SOD2 expression levels has in pancreatic carcinoma cell lines, since they have decreased expression of SOD2 resulting in increased proliferation rates.

One mechanism employed by cells for decreasing gene expression levels is hypermethylation of the promoter. We have recently shown that the sod 2 promoter is hypermethylated in 
Table 2 Identification of binding sites in the promoters of SOD2regulated genes for transcription factors showing altered DNA binding in MIA-PaCa2 cells with modulated SOD2

\section{Transcription}

factor module Gene promoters containing modules

\begin{tabular}{|c|c|}
\hline API_CEBP & wwox \\
\hline CEBP_STAT & wwox, fem lb \\
\hline SPI_ETS & $\begin{array}{l}\text { wwox, tbx22, man lal, suv39h2, tbcldl 4, efhd I, arhgap 24, grik5, } \\
\text { prkacb, gphn, abi2, jph4 (ap |g2), kcnabl, kcnip3 (csen), fndc3a, } \\
\text { aspa, mamdc2 }\end{array}$ \\
\hline ETS_CEBP & atp Ia2, prkacb, fndc3a \\
\hline EGR_SPI & $\begin{array}{l}\text { fstl5, fem Ib, man Ial, tbcldI 4, efhd I, arghap24, prkacb, abi2, } \\
\text { jph4 (ap Ig2), kcnip3 (csen), fndc3a }\end{array}$ \\
\hline CAAT_SPI & rorl, manlal \\
\hline CEBP_SPI & man Ial, prkacb, kcnabl \\
\hline BRN_p53 & prkacb, ugt2b7 \\
\hline BRN_RORA & kcnabl, kcnip3 (csen) \\
\hline CP2_SPI & $h b b$, ceacam4 \\
\hline SPI_CREB & sponl 1 \\
\hline
\end{tabular}

SOD2 = manganese superoxide dismutase. Binding sites for SOD2-regulated transcription factors in the promoters of SOD2-regulated genes. The promoters of the genes determined to be regulated by SOD2 (Figure 3) were identified using Genomatix's Gene2Promoter database (www.genomatix.de). Once identified, the promoters were mapped for transcription factor binding sites using Matlnspector (Genomatix, Ann Arbor, MI, USA). This table shows all the SOD2-regulated transcription factor (listed in this table) binding sites in the promoters of the SOD2regulated genes.

multiple myeloma (Hodge et al, 2005a), so we sought to determine if hypermethylation may be an explanation for the decreased expression of SOD2 observed in pancreatic carcinoma cell lines. Through sodium bisulphite sequencing, we show that the promoter of $\operatorname{sod} 2$ is hypermethylated in several cell lines (Figure 1), including MIA-PaCa2. Furthermore, the expression of SOD2 was increased following treatment of the cells with the methyltransferase inhibitor zebularine. The role of hypermethylation in the silencing of sod 2 in primary pancreatic carcinoma needs to be determined, but our initial studies using the pancreatic carcinoma cell lines indicate a possible role for hypermethylation.

Decreased expression of SOD2 would, theoretically, render the cells less capable of eliminating ROS that may build up in the cell. The decreased ability of cancer cells to eliminate ROS represents an Achille's heel that can be exploited to induce apoptosis of these cells. Indeed, cells with lower expression of SOD2 are more sensitive to the oxidative burst agent, 2ME2 (Figure 2). Therefore, for carcinomas in which there is decreased SOD2 activity, regardless of the mechanism, treatment with $2 \mathrm{ME} 2$ or other agents resulting in oxidative bursts may represent a potential treatment option.

While lowered SOD2 expression in itself might present treatment options for pancreatic carcinoma, the furthered understanding of the consequences of altered SOD2 expression illuminates other mechanisms that could be involved in the pathology of pancreatic cancer, suggesting further treatments. To this end, we examined in an integrated proteomic and genomic manner the consequences of SOD2 expression in MIA-PaCa2 cells by both overexpression and knockdown of SOD2. Using this approach, we have identified the genes that are regulated by SOD2 as well as the transcription factors whose binding activities are altered in response to SOD2 expression. The genes identified in Figure 3 function in diverse cellular processes, including calcium binding, steroid metabolism, and cell adhesion. Calcium binding is the most represented function according to Gene Ontology terms and is affected by the expression of five out of the 32 genes shown in Figure 3 (fstl5, man1a1, efhd1, prkacb, and kcnip3). The regulation of calcium homeostasis is complex, but there is evidence that ROS and calcium signalling pathways interact (reviewed by Yan et al, 2006). The identification of the transcriptional regulation of several genes involved in calcium regulation suggests that there may be an even greater interplay between these two signalling molecules and bears further investigation.

We identified the transcription factors in MIA-PaCa2 that show altered binding activity in response to altered levels of SOD2 (Table 1). First, it is interesting to point out that nearly all of the transcription factors identified show decreased binding. Second, almost all of these transcription factors are also impacted by calcium signalling. While the genes that are regulated by SOD2 expression could be regulated in some other manner, we sought to determine if there was overlap in the transcription factors identified as having SOD2-altered binding activities and the genes identified as being SOD2-regulated. In order to do this, we first identified the potential promoters of these genes using Genomatix's Gene2Promoter database. Once identified we used Genomatix's MatInspector to determine the transcription factor binding modules. More than $80 \%$ of the promoters searched contained binding sites for at least one of the regulated transcription factors. The identification of binding sites for SOD2-regulated transcription factors in the promoters of regulated genes (Table 2) strengthens the use of computer analysis to begin to identify signalling networks that may be at play in the cells studied.

We have shown that altered levels of SOD2 reveal a sensitivity to oxidative stress created by $2 \mathrm{ME} 2$ treatment and result in perturbations in the binding activities of several transcription factors, decreased phosphorylation of VEGFR2, and the alteration of the expression of several genes. The ability to determine both the genomic and proteomic events that change as a result of SOD2 expression, and therefore the redox status of the cell, provides us with a wealth of complex information. We have begun to understand the molecular consequences of altered SOD2 activity in pancreatic cancer and, furthermore, we have determined that the lack of SOD2 expression may result in a therapeutic advantage.

\section{ACKNOWLEDGEMENTS}

This publication has been funded in part with Federal funds from the National Cancer Institute, National Institutes of Health, under contract no. N01-CO-12400. This research was supported in part by the Intramural Research Program of the NIH, National Cancer Institute, Center for Cancer Research. The content of this publication does not necessarily reflect the views or policies of the Department of Health and Human Services, nor does mention of trade names, commercial products, or organisations imply endorsement by the US Government.

Supplementary Information accompanies the paper on British Journal of Cancer website (http://www.nature.com/bjc)

\section{REFERENCES}

Best CJ, Gillespie JW, Yi Y, Chandramouli GV, Perlmutter MA, Gathright Y, Erickson HS, Georgevich L, Tangrea MA, Duray PH, Gonzalez S, Velasco A, Linehan WM, Matusik RJ, Price DK, Figg WD, Emmert-Buck MR, Chuaqui RF (2005) Molecular alterations in primary prostate cancer after androgen ablation therapy. Clin Cancer Res 11: $6823-6834$

Brummelkamp TR, Bernards R, Agami R (2002) Stable suppression of tumorigenicity by virus-mediated RNA interference. Cancer Cell 2: 243-247 
Cadenas E (1989) Biochemistry of oxygen toxicity. Annu Rev Biochem 58: $79-110$

Chauhan D, Li G, Sattler M, Podar K, Mitsiades C, Mitsiades N, Munshi N, Hideshima T, Anderson KC (2003) Superoxide-dependent and -independent mitochondrial signaling during apoptosis in multiple myeloma cells. Oncogene 22: 6296-6300

Cullen JJ, Weydert C, Hinkhouse MM, Ritchie J, Domann FE, Spitz D, Oberley LW (2003) The role of manganese superoxide dismutase in the growth of pancreatic adenocarcinoma. Cancer Res 63: 1297-1303

Driscoll JS, Marquez VE, Plowman J, Liu PS, Kelley JA, Barchi Jr JJ (1991) Antitumor properties of 2(1H)-pyrimidinone riboside (zebularine) and its fluorinated analogues. I Med Chem 34: 3280-3284

Gonzalez-Pacheco FR, Deudero JJ, Castellanos MC, Castilla MA, varezArroyo MV, Yague S, Caramelo C (2006) Mechanisms of endothelial response to oxidative aggression: protective role of autologous VEGF and induction of VEGFR2 by $\mathrm{H}_{2} \mathrm{O}_{2}$. Am J Physiol Heart Circ Physiol 291: $\mathrm{H} 1395$ - $\mathrm{H} 1401$

Hodge DR, Peng B, Pompeia C, Thomas S, Cho E, Clausen PA, Marquez VE, Farrar WL (2005a) Epigenetic silencing of manganese superoxide dismutase (SOD-2) in KAS 6/1 human multiple myeloma cells increases cell proliferation. Cancer Biol Ther 4: 585-592

Hodge DR, Xiao W, Peng B, Cherry JC, Munroe DJ, Farrar WL (2005b) Enforced expression of superoxide dismutase 2/manganese superoxide dismutase disrupts autocrine interleukin-6 stimulation in human multiple myeloma cells and enhances dexamethasone-induced apoptosis. Cancer Res 65: 6255-6263

Hussain SP, Hofseth LJ, Harris CC (2003) Radical causes of cancer. Nat Rev Cancer 3: $276-285$

Kiningham KK, St Clair DK (1997) Overexpression of manganese superoxide dismutase selectively modulates the activity of Jun-associated transcription factors in fibrosarcoma cells. Cancer Res 57: 5265-5271

Li JJ, Colburn NH, Oberley LW (1998a) Maspin gene expression in tumor suppression induced by overexpressing manganese-containing superoxide dismutase cDNA in human breast cancer cells. Carcinogenesis 19: 833 - 839

Li N, Oberley TD, Oberley LW, Zhong W (1998b) Overexpression of manganese superoxide dismutase in DU145 human prostate carcinoma cells has multiple effects on cell phenotype. Prostate 35: $221-233$

Lin X, Tascilar M, Lee WH, Vles WJ, Lee BH, Veeraswamy R, Asgari K, Freije D, van RB, Gage WR, Bova GS, Isaacs WB, Brooks JD, DeWeese TL, De Marzo AM, Nelson WG (2001) GSTP1 CpG island hypermethylation is responsible for the absence of GSTP1 expression in human prostate cancer cells. Am J Pathol 159: $1815-1826$

Liu J, Hinkhouse MM, Sun W, Weydert CJ, Ritchie JM, Oberley LW, Cullen JJ (2004) Redox regulation of pancreatic cancer cell growth: role of glutathione peroxidase in the suppression of the malignant phenotype. Hum Gene Ther 15: 239-250

Liu R, Oberley TD, Oberley LW (1997) Transfection and expression of MnSOD cDNA decreases tumor malignancy of human oral squamous carcinoma SCC-25 cells. Hum Gene Ther 8: 585-595

Melov S (2000) Mitochondrial oxidative stress. Physiologic consequences and potential for a role in aging. Ann N Y Acad Sci 908: 219-225

Nelson WG, De Marzo AM, DeWeese TL (2001) The molecular pathogenesis of prostate cancer: implications for prostate cancer prevention. Urology 57: $39-45$

Ough M, Lewis A, Zhang Y, Hinkhouse MM, Ritchie JM, Oberley LW, Cullen JJ (2004) Inhibition of cell growth by overexpression of manganese superoxide dismutase (MnSOD) in human pancreatic carcinoma. Free Radic Res 38: 1223 - 1233

Paweletz CP, Charboneau L, Bichsel VE, Simone NL, Chen T, Gillespie JW, Emmert-Buck MR, Roth MJ, Petricoin III EF, Liotta LA (2001) Reverse phase protein microarrays which capture disease progression show activation of pro-survival pathways at the cancer invasion front. Oncogene 20: 1981 - 1989

Qanungo S, Basu A, Das M, Haldar S (2002) 2-Methoxyestradiol induces mitochondria dependent apoptotic signaling in pancreatic cancer cells. Oncogene 21: $4149-4157$

Schumacher G, Kataoka M, Roth JA, Mukhopadhyay T (1999) Potent antitumor activity of 2-methoxyestradiol in human pancreatic cancer cell lines. Clin Cancer Res 5: 493-499

Soini Y, Vakkala M, Kahlos K, Paakko P, Kinnula V (2001) MnSOD expression is less frequent in tumour cells of invasive breast carcinomas than in in situ carcinomas or non-neoplastic breast epithelial cells. J Pathol 195: $156-162$

Su SB, Motoo Y, Xie MJ, Mouri H, Asayama K, Sawabu N (2002) Superoxide dismutase is induced during rat pancreatic acinar cell injury. Pancreas 24: $146-152$

Van Remmen H, Ikeno Y, Hamilton M, Pahlavani M, Wolf N, Thorpe SR, Alderson NL, Baynes JW, Epstein CJ, Huang TT, Nelson J, Strong R, Richardson A (2003) Life-long reduction in MnSOD activity results in increased DNA damage and higher incidence of cancer but does not accelerate aging. Physiol Genomics 16: 29-37

Venkataraman S, Jiang X, Weydert C, Zhang Y, Zhang HJ, Goswami PC, Ritchie JM, Oberley LW, Buettner GR (2005) Manganese superoxide dismutase overexpression inhibits the growth of androgen-independent prostate cancer cells. Oncogene 24: 77-89

Weydert C, Roling B, Liu J, Hinkhouse MM, Ritchie JM, Oberley LW, Cullen JJ (2003) Suppression of the malignant phenotype in human pancreatic cancer cells by the overexpression of manganese superoxide dismutase. Mol Cancer Ther 2: $361-369$

Xu Y, Krishnan A, Wan XS, Majima H, Yeh CC, Ludewig G, Kasarskis EJ, St Clair DK (1999) Mutations in the promoter reveal a cause for the reduced expression of the human manganese superoxide dismutase gene in cancer cells. Oncogene 18: $93-102$

Yan Y, Wei CL, Zhang WR, Cheng HP, Liu J (2006) Cross-talk between calcium and reactive oxygen species signaling. Acta Pharmacol Sin 27: $821-826$

Zhao Y, Xue Y, Oberley TD, Kiningham KK, Lin SM, Yen HC, Majima H, Hines J, St Clair D (2001) Overexpression of manganese superoxide dismutase suppresses tumor formation by modulation of activator protein-1 signaling in a multistage skin carcinogenesis model. Cancer Res 61: $6082-6088$

Zhong W, Oberley LW, Oberley TD, St Clair DK (1997) Suppression of the malignant phenotype of human glioma cells by overexpression of manganese superoxide dismutase. Oncogene 14: $481-490$

Zhong W, Yan T, Webber MM, Oberley TD (2004) Alteration of cellular phenotype and responses to oxidative stress by manganese superoxide dismutase and a superoxide dismutase mimic in RWPE-2 human prostate adenocarcinoma cells. Antioxid Redox Signal 6: $513-522$

Zhou L, Cheng X, Connolly BA, Dickman MJ, Hurd PJ, Hornby DP (2002) Zebularine: a novel DNA methylation inhibitor that forms a covalent complex with DNA methyltransferases. J Mol Biol 321: $591-599$ 